\title{
Combining Electromagnetic and Gravitational-Wave Constraints on Neutron-Star Masses and Radii
}

\author{
Mohammad Al-Mamun ${ }^{1}$, Andrew W. Steiner ${ }^{1,2}$, Joonas Nättilä ${ }^{3,4}$, Jacob Lange ${ }^{5}$, Richard \\ O'Shaughnessy $^{5}$, Ingo Tews ${ }^{6}$, Stefano Gandolfi ${ }^{6}$, Craig Heinke ${ }^{7}$, and Sophia Han ${ }^{8,9}$ \\ ${ }^{1}$ Department of Physics and Astronomy, University of Tennessee, Knoxville, TN 37996, USA \\ ${ }^{2}$ Physics Division, Oak Ridge National Laboratory, Oak Ridge, TN 37831, USA \\ ${ }^{3}$ Physics Department and Columbia Astrophysics Laboratory, \\ Columbia University, 538 West 120th Street, New York, NY 10027, USA \\ ${ }^{4}$ Center for Computational Astrophysics, Flatiron Institute, 162 Fifth Avenue, New York, NY 10010, USA \\ ${ }_{5}^{5}$ Rochester Institute of Technology, 85 Lomb Memorial Drive, Rochester, NY 14623, USA \\ ${ }^{6}$ Theoretical Division, Los Alamos National Laboratory, Los Alamos, NM 87545, USA \\ 7 Department of Physics, CCIS 4-183, University of Alberta, Edmonton, AB, T6G 2E1, Canada \\ ${ }^{8}$ Department of Physics, University of California, Berkeley, CA 94720, USA and \\ 9 Department of Physics and Astronomy, Ohio University, Athens, OH 45701, USA
}

\begin{abstract}
We perform a joint Bayesian inference of neutron-star mass and radius constraints based on GW170817, observations of quiescent low-mass X-ray binaries (QLMXBs), photospheric radius expansion X-ray bursts (PREs), and X-ray timing observations of J0030+0451. With this data set, the form of the prior distribution still has an impact on the posterior mass-radius (MR) curves and equation of state (EOS), but this impact is smaller than recently obtained when considering QLMXBs alone. We analyze the consistency of the electromagnetic data by including an "intrinsic scattering" contribution to the uncertainties, and find only a slight broadening of the posteriors. This suggests that the gravitational-wave and electromagnetic observations of neutron-star structure are providing a consistent picture of the neutron-star mass-radius curve and the EOS.
\end{abstract}

PACS numbers: 97.60.Jd, 95.30.Cq, 26.60.-c

The idea that neutron stars (NSs) might be useful in determining the equation of state (EOS) of dense matter [1] precedes the discovery of the first NS in 1967 [2] by almost a decade. Until recently, the strongest observational constraints on the EOS came from NS mass measurements. These mass measurements all lay in a narrow range around $1.4 \mathrm{M}_{\odot}$ [3] until the last decade, when NSs with masses near $2.0 \mathrm{M}_{\odot}$ were discovered [48]. Measurements of NS radii, on the other hand, have been plagued with various systematic uncertainties [9]. The past decade has seen an increasing number of observations which constrain both the NS mass and radius with better-controlled systematic uncertainties, providing stronger EOS constraints. Quiescent low-mass Xray binaries [10] ("QLMXBs"), NSs which exhibit photospheric radius expansion X-ray bursts [11] ("PREs"), and nearby isolated NSs $[12,13]$ all have been used to provide mass and radius measurements (see e.g. Refs. [14, 15] for recent reviews). Importantly, these measurements rely on data from various different instruments and connect the radius to the actual observables using different theoretical assumptions. These in turn all yield different underlying systematics. Finally, the recent observations of gravitational waves $(\mathrm{GW})$ from binary NS mergers (GW170817 and GW190425) [16-18] by the LIGO Scientific- and Virgo collaborations (LVC) or Xray observations of J0030+0451 by the NICER collaboration $[19,20]$ provide additional information on the EOS.

There are several recent works which analyze the data from GW170817 and/or GW190425 as well as NICER and its implication on the NS EOS [21-40], but very few (e.g., Ref. [31, 41]) directly combine the GW data with constraints on NS radii from QLMXBs and PREs. However, these additional observational sources add valuable information. Ref. [42] used QLMXBs and PREs observations to predict the NS tidal deformability which would be inferred from GW observations. They predicted the dimensionless tidal deformability of a $1.4 \mathrm{M}_{\odot} \mathrm{NS}$, $\Lambda_{M=1.4}$, was between 130 and 460 to $95 \%$ confidence. The recent analysis of GW 170817 [17] by the LVC found $\Lambda_{M=1.4} \in[70,580]$, to $90 \%$ confidence, matching the prediction to within errors.

In this letter, we present a Bayesian inference of the NS structure data, including both GW data as well as data from electromagnetic observation of QLMXBs and PREs, using less restrictive assumptions than made in previous works.

We build upon the method first described in Ref. [43, 44] (see also Ref. [45]), reviewed in Ref. [46], and detailed in the Supplemental Material. Ref. [47] first demonstrated that the choice of EOS parameterization has a significant impact on both the posterior mass-radius relation and the EOS, see also Ref. [48]. To estimate the impact of that choice, here as in Ref. [49], our prior distribution is built on two EOS parameterizations: (a) one which uses three polytropes (referred to as "3P") and (b) one which uses four line segments in the space of pressure vs. energy density (referred to as " $4 \mathrm{~L}$ "). The latter parametrization has a stronger preference for strong phase transitions (regions where the pressure is nearly 
independent of the energy density). We use these parameterizations because both of them are physically reasonable, yet they give qualitatively different mass-radius curves. This allows us to study how the prior choice creates additional uncertainty in our results.

At each point in our EOS parameter spaces, we solve the Tolman-Oppenheimer-Volkov (TOV) equations [50, 51], compute the moment of inertia as a function of the central pressure, and use the Yagi-Yunes (YY) correlation [52] (as formulated in Ref. [53]) to compute the tidal deformability as a function of central pressure. This method of computing the tidal deformability is much faster than a direct computation, and while deviations from the exact result of up to $10 \%$ are possible [54, 55], the correlation is accurate to within a few percent for the typical EOS in our prior and posterior distributions. We construct the conditional probability for QLMXBs as in Ref. [49] and generalize it to include 3 PRE X-ray bursting sources $[56,57]$. We also include the LIGO constraints on $\tilde{\Lambda}$ from GW170817, using the SEOBNRv4T model for binary NS inspiral assuming low compact object spins [58]. GW observations are incorporated by tabulating and interpolating a marginal likelihood versus the masses for each object and the combined tidal deformability. Marginalization is performed via RIFT [59] over all extrinsic parameters and our fiducial low-spin prior assumptions. Finally, we also include mass and radius constraints on J0030+0451 from the NICER instrument $[19,20]$. We reject all EOSs which are acausal or imply a maximum NS mass less than $2 \mathrm{M}_{\odot}$. The mass cutoff does have a small uncertainty, but this uncertainty does not qualitatively impact our results. Also, we could have chosen to replace one of the high-density EOS parameters with $M_{\max }$, but since neither the high-density part of the EOS nor the maximum mass is well-known, this prior choice is not necessarily better (or worse) than ours.

While it is often helpful to directly model systematic uncertainties, as was partially done in earlier works [49, $56,57]$, these estimations require detailed models for the uncertainties which may not be perfect. Systematic uncertainties may result in intrinsic scattering (IS) which we model by convolving the probability distribution $\mathcal{D}(R, M)$ for each star with a Gaussian kernel. This addition of an extra uncertainty to the observations allows us to quantify the possible level of systematic bias present in each measurement: the value of each IS parameter is expected to increase until the full dataset used in the inference is self-consistent. We emphasize that there is no reason to believe that a Gaussian is necessarily the correct distribution for additional unknown systematics, but we believe it is a reasonable first guess. Our approach is based on the intrinsic scattering parameter from Ref. [57] which was applied to the X-ray spectrum rather than the mass and radius constraints. The details of this procedure and the Markov Chain Monte Carlo (MCMC)

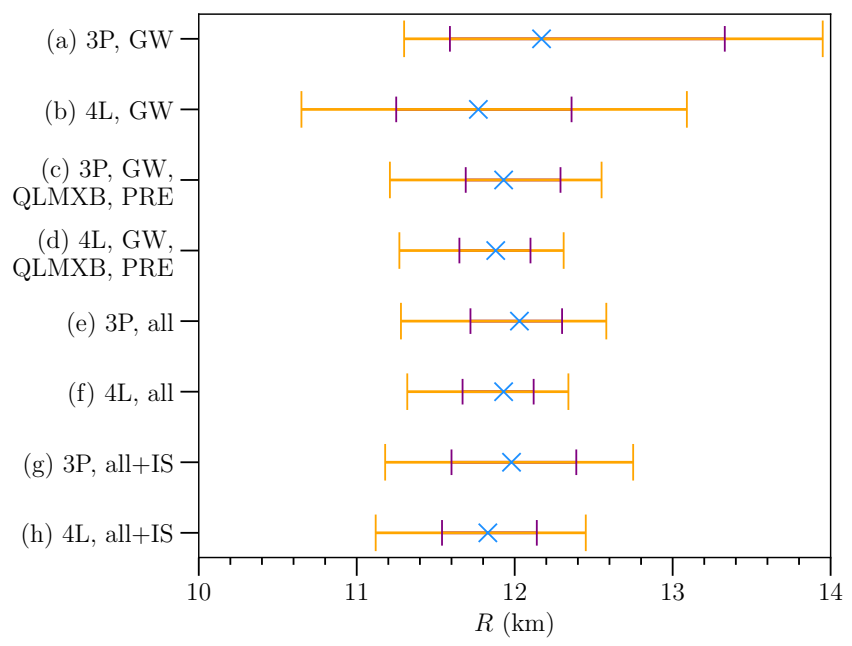

FIG. 1. Radius measured for a $1.4 \mathrm{M}_{\odot} \mathrm{NS}$ in different models. Blue crosses indicate the median points, while the purple and orange bars represent the $68 \%$ and $95 \%$ credible intervals.

method are given in the Supplemental Material.

We argue that neither of the two EOS parameterizations are more or less motivated by quantum chromodynamics (QCD), and thus we assign them equal prior probability. We present the posteriors for these two EOS parameterizations, $3 \mathrm{P}$ and $4 \mathrm{~L}$, separately to make the impact of a different EOS parameterization clear. We also present four different data sets: (i) GW170817 only (GW), (ii) GW170817+QLMXBs+PREs (GW, QLMXB, PRE), (iii) GW170817+QLMXBs+PREs+NICER (all), and (iv) GW170817+QLMXBs+PREs+NICER with an additional intrinsic scattering parameter added to each of the QLMXBs, PREs and NICER (all+IS). We thus performed 8 different sets of simulations.

Posteriors for the radius of a 1.4 solar mass NS, $R_{1.4}$, are summarized in Figure 1. As might be expected, the limits for the GW data alone [(a) and (b)] are the least constraining. The $95 \%$ credible intervals are $11.3-13.9 \mathrm{~km}(3 \mathrm{P})$ and $10.7-13.1 \mathrm{~km}(4 \mathrm{~L})$. Choosing a line segment-based EOS prior decreases the lower (upper) radius limit by $0.5(1) \mathrm{km}$. The next four bar plots in the figure [(c) - (f)] show that the range shrinks significantly when adding the EM observations without IS. In addition, once the EM observations are added, these constraints are less sensitive to the EOS prior than the QLMXB observations alone [49]. Including the IS contribution $[(\mathrm{g})$ and $(\mathrm{h})]$ slightly broadens the constraints coming from the EM data.

Figure 2 shows the posterior distributions for the NS radius as a function of the gravitational mass. The shape of the M-R curve is more sensitive to the EOS prior than the radius of a 1.4 solar mass NS alone. The $4 \mathrm{~L}$ EOS prior, because of the potential for phase transitions to 


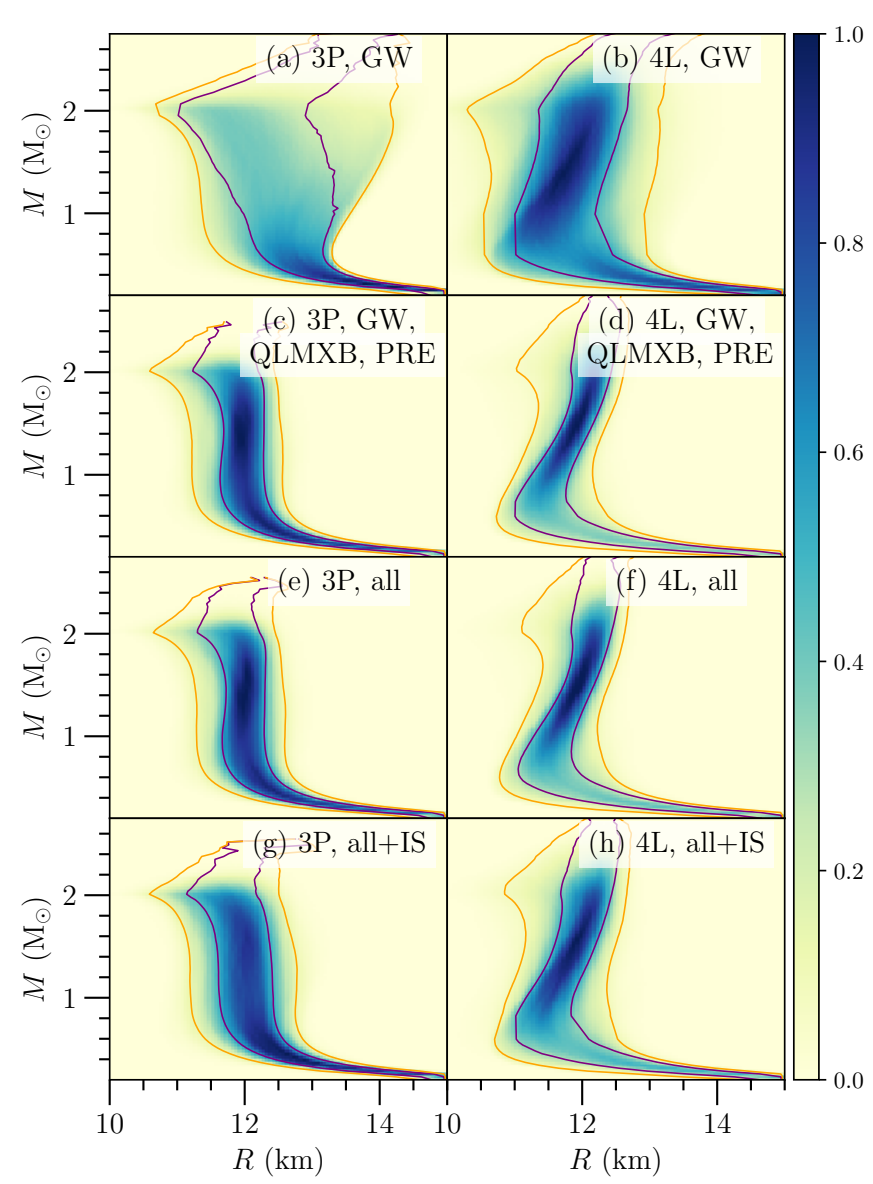

FIG. 2. Posterior distributions for the NS radius as a function of the gravitational mass. Left panels are constructed with the " $3 \mathrm{P}$ " EOS and right panels with the " $4 \mathrm{~L}$ " EOS. Different rows refer to different data selections.

modify the EOS at low densities, produces smaller radii for low-mass stars and larger radii for high-mass stars. This distinction may be particularly important in light of a possible $2.6 \mathrm{M}_{\odot}$ NS in GW190814 [60-62].

Figure 3 shows the posteriors for the pressure as a function of the energy density. The combination of the EM and GW data strongly constrains the pressure until about $400-500 \mathrm{MeV} / \mathrm{fm}^{3}$. In order to more easily compare the pressures between different models or data sets, Figure 4 shows an alternate version where, for each energy density, all 8 panels are rescaled and shifted by the same linear transformation which ensures that the $95 \%$ credible intervals for panel (a) lie exactly at 0 and 1 . Thus, for panels (b) through (h), the orange dashed curves show the change in pressure of different models relative to that in panel (a). For reference, the energy density at nuclear saturation is $\epsilon_{0} \approx 150 \mathrm{MeV} / \mathrm{fm}^{3}$. The red dot-dashed curves show the probability that the central energy density of the maximum mass NS is smaller than the energy density from the $\mathrm{x}$-axis. Most EOSs suggested by the data imply the central energy density is between about 900 and $1200 \mathrm{MeV} / \mathrm{fm}^{3}$. In the 3P model, the EM data

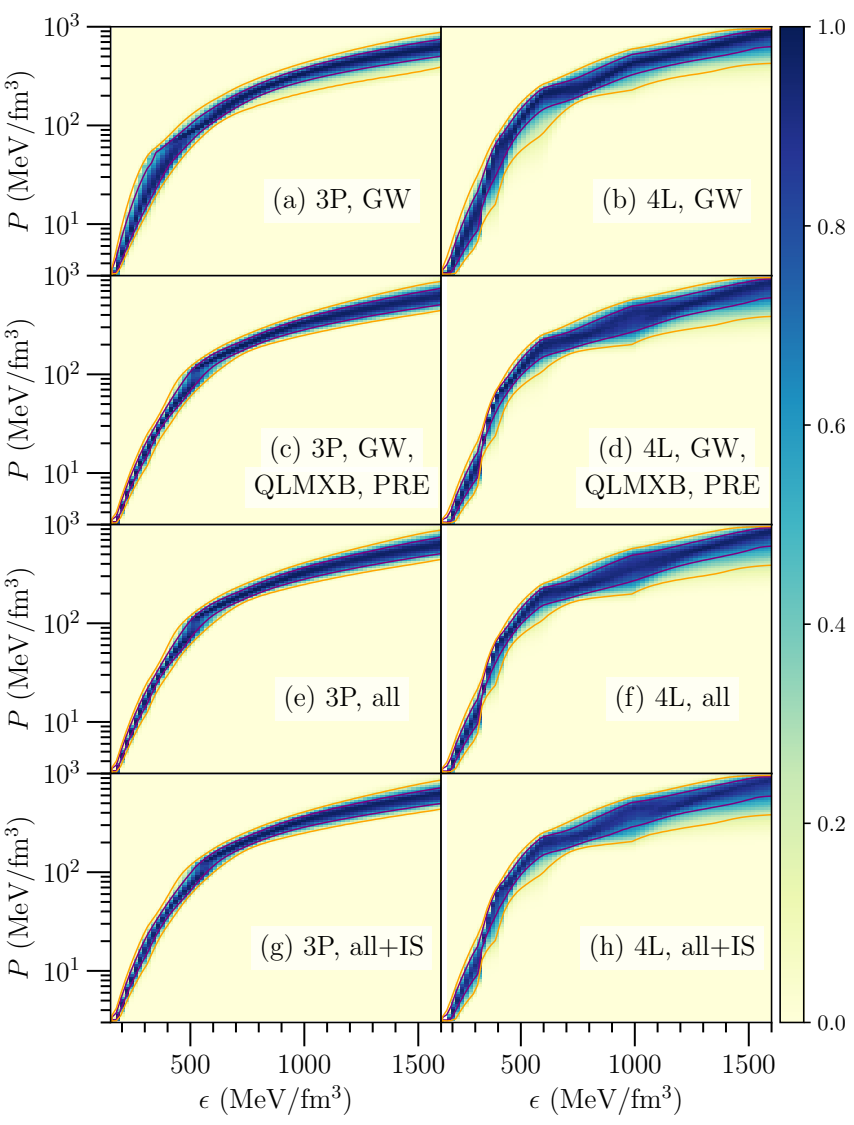

FIG. 3. Posterior distributions for the pressure as a function of the energy density. Left panels are constructed with the " $3 \mathrm{P}$ " EOS and right panels with the " $4 \mathrm{~L}$ " EOS. Different rows refer to different data selections.

suggests a smaller pressure for small energy densities and almost unchanged at higher densities. In the $4 \mathrm{~L}$ model, the effect is more dramatic: a smaller pressure at low densities is compensated for by an increase in the pressure at higher densities. For $\epsilon>1200 \mathrm{MeV} / \mathrm{fm}^{3}$, it is unlikely the data is strongly constraining the EOS, i.e. the EOS is strongly impacted by the prior distribution.

We compare our results to several other previous works in Table I. Our results on $R_{1.4}$ with the GW data alone are consistent with previous works which include only limited information from NS radius constraints [21, 23, 27-30,39]. The variation in these results across the various references is consistent with our finding that these posterior distributions depend on the EOS prior distribution (as well as on the other details of the analysis).

Other works find, as we do, that the radius constraints are tighter when the EM data is included. Our results which include IS suggest (but do not definitively prove) that this result is not due to systematic uncertainties which are artificially constraining NS radii.

Gravitational wave observations have suggested other possible more indirect constraints on the EOS, and we 


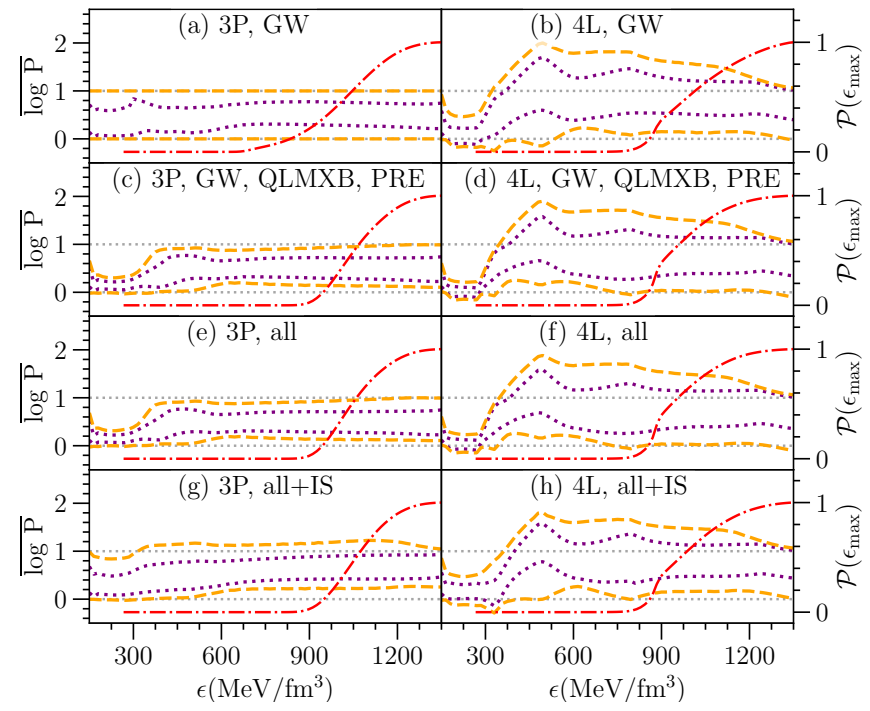

FIG. 4. The $68 \%$ (purple dotted) and 95\% (orange dashed) credible intervals for the pressure as a function of the energy density. All intervals are modified with the same linear transformation to ensure that the upper and lower $95 \%$ intervals in panel (a) are always at 0 and 1 . The right-hand y axis (red dot-dashed line) shows the posterior probability that the central energy density is smaller than the value on the $\mathrm{x}$ axis.

summarize the impact of some of these constraints have on the radius of a $1.4 \mathrm{M}_{\odot} \mathrm{NS}$ in Table II by applying them to our "all+IS" posteriors. Refs. [29,63] found that GW170817 implied a lower limit on $\tilde{\Lambda}>300$, because EOSs with smaller values of $\tilde{\Lambda}$ do not create a sufficiently massive accretion disk to the observed kilonova. This constraint may be optimistic (e.g. see Ref. [64]) (and see also a different perspective in Ref. [65]), but it has a relatively low impact. It increases the lower limit on the $95 \%$ confidence limit for $R_{1.4}$ by about $0.2 \mathrm{~km}$. Ref. [66] found that $M_{\max } \leq 2.17 \mathrm{M}_{\odot}$ was required to ensure that no short-lived hypermassive NS (which was not observed) was present in GW170817. We find that this constraint has only a weak impact on our posterior distributions. The maximum mass is weakly correlated by the radius of a 1.4 solar mass NS, as shown the Supplemental Material, so when we decrease the typical maximum mass we also slightly decrease the typical radius. Finally, GW190814 implied the merger of a $2.6 \mathrm{M}_{\odot}$ object with a more massive black hole. Our MCMC simulation for the 3P model generated no configurations with maximum masses this large. This does not necessarily mean that $3 \mathrm{P}$ parameter sets with large maximum masses do not exist, but they do appear highly improbable. In the $4 \mathrm{~L}$ model, increasing $M_{\max }$ to $2.6 \mathrm{M}_{\odot}$ increases the $95 \%$ lower limit for the radius by $0.5 \mathrm{~km}$. For example, in Table II, changing the $M_{\max }$ value in $4 \mathrm{~L}$ models increased $R$ lower limit of $95 \%$ C.I. from $10.98 \mathrm{~km}$ to $11.47 \mathrm{~km}$ for a $1.4 M_{\odot}$ NS.

M.A. was supported by NSF grant AST 1909490. A.W.S. was supported by NSF grant PHY 1554876, by

\begin{tabular}{c|cl}
\hline Reference & $\mathrm{R}_{1.4}$ & C.I. Source \\
\hline$[17]$ & {$[10.5,13.3]$} & $90 \% \mathrm{GW}$ \\
{$[21]$} & {$[9.9,13.6]$} & $90 \% \mathrm{GW}$ \\
{$[22]$} & $<13.6$ & $90 \% \mathrm{GW}$ \\
{$[23]$} & {$[9.4,12.8]$} & $90 \% \mathrm{GW}$ \\
{$[27]$} & {$[9.8,13.2]^{\mathrm{a}}$} & $90 \% \mathrm{GW}$ \\
{$[36]$} & {$[10.36,12.78]$} & $90 \% \mathrm{GW}$ \\
Model "a" & {$[11.30,13.95]$} & $95 \% \mathrm{GW}$ \\
Model "b" & {$[10.65,13.09]$} & $95 \% \mathrm{GW}$ \\
{$[28]$} & {$[8.9,13.2]$} & $90 \% \mathrm{GW}$, merger remnant \\
{$[29]$} & {$[11.4,13.2]$} & $90 \% \mathrm{GW}$, merger remnant \\
{$[30]$} & {$[10.4,11.9]$} & $90 \% \mathrm{GW}$, merger remnant \\
{$[31]$} & {$[11.98,12.76]$} & $90 \% \mathrm{GW}$, QLMXB \\
{$[32]$} & {$[10.5,11.8]$} & $90 \% \mathrm{GW}$, QLMXB \\
{$[33]$} & {$[10.94,12.72]$} & $90 \% \mathrm{GWs}$, NICER \\
{$[34,35]$} & {$[10.85,13.41]$} & $90 \%$ GWs, NICER \\
{$[36]$} & {$[11.91,13.25]$} & $90 \%$ GW, NICER \\
{$[37]$} & {$[11.3,13.3]$} & $90 \%$ GW, NICER \\
{$[41]$} & {$[12,13]$} & $90 \%$ GWs, NICER \\
{$[41]$} & {$[10.0,11.5]$} & $90 \%$ GWs, QLMXB, PRE \\
Model "c" & {$[11.21,12.55]$} & $95 \%$ GW, QLMXB, PRE \\
Model "e" & {$[11.28,12.58]$} & $95 \%$ GW, QLMXB, PRE, NICER \\
\hline a Radius measurement for the primary NS of the merger event \\
b GWs referred to the joint analysis of GW170817 and GW190425
\end{tabular}

TABLE I. A comparison of our posterior distributions for the radius of a $1.4 \mathrm{M}_{\odot} \mathrm{NS}$ in comparison to other results obtained in the literature.

\begin{tabular}{c|ccccc}
\hline Model \& constraints & $-2 \sigma$ & $-1 \sigma$ & med. & $+1 \sigma$ & $+2 \sigma$ \\
\hline 3P, all+IS & 11.18 & 11.6 & 11.98 & 12.39 & 12.75 \\
4L, all $+\mathrm{IS}$ & 11.12 & 11.54 & 11.83 & 12.14 & 12.45 \\
3P, all+IS $(\tilde{\Lambda}>300)$ & 11.33 & 11.63 & 11.98 & 12.37 & 12.71 \\
4L, all $+\mathrm{IS}(\tilde{\Lambda}>300)$ & 11.28 & 11.56 & 11.83 & 12.12 & 12.40 \\
$3 \mathrm{P}$, all $+\mathrm{IS}\left(M_{\max }<2.17 \mathrm{M}_{\odot}\right)$ & 11.15 & 11.54 & 11.98 & 12.31 & 12.72 \\
4L, all+IS $\left(M_{\max }<2.17 \mathrm{M}_{\odot}\right)$ & 10.98 & 11.43 & 11.88 & 12.13 & 12.46 \\
$4 \mathrm{~L}$, all $+\mathrm{IS}\left(M_{\max }>2.6 \mathrm{M}_{\odot}\right)$ & 11.47 & 11.76 & 11.98 & 12.19 & 12.42 \\
\hline
\end{tabular}

TABLE II. The $1 \sigma$ and $2 \sigma$ confidence limits with the median for the radius of $1.4 \mathrm{M}_{\odot} \mathrm{NS}$ in $\mathrm{km}$ with applied constraints on intrinsic scattering models.

the U.S. DOE Office of Nuclear Physics, Nordic Institute for Theoretical Physics (NORDITA), and the University of Turku. J.L. and R.O.S. were supported by NSF grants PHY 1707965 and AST 1909534. I.T. and S.G. were supported by the U.S. Department of Energy, Office of Science, Office of Nuclear Physics, under contract No. DE-AC52-06NA25396, by the NUCLEI SciDAC program, and by the LDRD program at LANL. S.G. was also supported by the DOE Early Career research Program. C.H. is supported by NSERC Discovery Grant RGPIN-2016-04602, and a Discovery Accelerator Supple- 
ment. S.H. is supported by the National Science Foundation, Grant PHY-1630782, and the Heising-Simons Foundation, Grant 2017-228. This work used the Extreme Science and Engineering Discovery Environment (XSEDE) allocation PHY 170048 and PHY 180052 supported by NSF grant number ACI-1548562. The open-source code for this work [67] is built upon $\mathrm{O}_{2} \mathrm{scl}$ [68], GSL, HDF5, FFTW [69], and matplotlib.

[1] A. G. Cameron, Astrophys. J. 130, 884 (1959), URL https://doi.org/10.1086/146780.

[2] A. Hewish, S. J. Bell, J. D. H. Pilkington, P. F. Scott, and R. A. Collins, Nature 217 (1968), URL https:// doi.org/10.1038/217709a0.

[3] S. E. Thorsett and D. Chakrabarty, Astrophys. J. 512, 288 (1999), URL https://doi.org/10.1086/306742.

[4] Barziv, O., Kaper, L., Van Kerkwijk, M. H., Telting, J. H., and Van Paradijs, J., Astron. \& Astrophys. 377, 925 (2001), URL https://doi.org/10.1051/ 0004-6361:20011122.

[5] P. B. Demorest, T. Pennucci, S. M. Ransom, M. S. E. Roberts, and J. W. T. Hessels, Nature (London) 467, 1081 (2010), URL https://dx.doi.org/10.1038/ nature09466.

[6] M. H. van Kerkwijk, R. P. Breton, and S. R. Kulkarni, Astrophys. J. 728, 95 (2011), URL https://doi.org/ 10.1088/0004-637X/728/2/95.

[7] J. Antoniadis, P. C. C. Freire, N. Wex, T. M. Tauris, R. S. Lynch, M. H. van Kerkwijk, M. Kramer, C. Bassa, V. S. Dhillon, T. Driebe, et al., Science 340, 6131 (2013), URL https://doi.org/10.1126/science.1233232.

[8] H. T. Cromartie, E. Fonseca, S. M. Ransom, P. B. Demorest, Z. Arzoumanian, H. Blumer, P. R. Brook, M. E. DeCesar, T. Dolch, J. A. Ellis, et al., Nature Astron. 4, 72 (2020), URL https://doi.org/10.1038/ s41550-019-0880-2.

[9] J. M. Lattimer and M. Prakash, Physics Reports 442, 109 (2007), ISSN 0370-1573, the Hans Bethe Centennial Volume 1906-2006, URL https://doi.org/10.1016/j. physrep.2007.02.003.

[10] C. O. Heinke, G. B. Rybicki, R. Narayan, and J. E. Grindlay, Astrophys. J. 644, 1090 (2006), URL https: //doi.org/10.1086/503701.

[11] F. Özel, T. Güver, and D. Psaltis, Astrophys. J. 693, 1775 (2009), URL https://doi.org/10.1088/ 0004-637x/693/2/1775.

[12] J. A. Pons, F. M. Walter, J. M. Lattimer, M. Prakash, R. Neuhauser, and P.-h. An, Astrophys. J. 564, 981 (2002), URL https://doi.org/10.1086/324296.

[13] W. C. G. Ho and C. O. Heinke, Nature 462, 71 (2009), URL https://doi.org/0.1038/nature08525.

[14] J. M. Lattimer, Annu. Rev. Nucl. Part. Sci. 62, 485 (2012), URL https://doi.org/10.1146/ annurev-nucl-102711-095018.

[15] F. Ozel and P. Freire, Ann. Rev. Astron. Astrophys. 54, 401 (2016), URL https://doi.org/10.1146/ annurev-astro-081915-023322.

[16] LIGO Scientific Collab. and Virgo Collab., Phys. Rev. Lett. 119, 161101 (2017), URL https://doi.org/10.
1103/PhysRevLett.119.161101.

[17] LIGO Scientific Collab. and Virgo Collab., Phys. Rev. Lett. 121, 161101 (2018), URL https://doi.org/10. 1103/PhysRevLett.121.161101.

[18] B. Abbott et al. (LIGO Scientific, Virgo), Astrophys. J. Lett. 892, L3 (2020), URL https://doi.org/10.3847/ 2041-8213/ab75f5.

[19] T. E. Riley, A. L. Watts, S. Bogdanov, P. S. Ray, R. M. Ludlam, S. Guillot, Z. Arzoumanian, C. L. Baker, A. V. Bilous, D. Chakrabarty, et al., Astrophys. J. 887, L21 (2019), URL https://doi.org/10.3847/ 2041-8213/ab481c.

[20] M. C. Miller et al., Astrophys. J. 887, L24 (2019), URL https://doi.org/10.3847/2041-8213/ab50c5.

[21] E. Annala, T. Gorda, A. Kurkela, and A. Vuorinen, Phys. Rev. Lett. 120, 172703 (2018), URL https://doi.org/ 10.1103/PhysRevLett.120.172703.

[22] F. J. Fattoyev, J. Piekarewicz, and C. J. Horowitz, Phys. Rev. Lett. 120, 172702 (2018), URL https://doi.org/ 10.1103/PhysRevLett.120.172702.

[23] B. Kumar and P. Landry, Phys. Rev. D 99, 123026 (2019), URL https://doi.org/10.1103/PhysRevD.99. 123026.

[24] M. W. Coughlin et al., Mon. Not. Roy. Astron. Soc. 480, 3871 (2018), 1805.09371, URL https://doi.org/ $10.1093 / \mathrm{mnras} / \mathrm{sty} 2174$.

[25] M. W. Coughlin, T. Dietrich, B. Margalit, and B. D. Metzger, Mon. Not. Roy. Astron. Soc. 489, L91 (2019), 1812.04803, URL https://dx.doi.org/10. $1093 / \mathrm{mnrasl} / \mathrm{slz} 133$.

[26] I. Tews, J. Margueron, and S. Reddy, Phys. Rev. C 98, 045804 (2018), 1804.02783, URL https://doi.org/10. 1103/PhysRevC.98.045804.

[27] C. A. Raithel, Eur. Phys. J. A 55, 80 (2019), URL https : //doi.org/10.1140/epja/i2019-12759-5.

[28] S. De, D. Finstad, J. M. Lattimer, D. A. Brown, E. Berger, and C. M. Biwer, Phys. Rev. Lett. 121, 091102 (2018), URL https://doi.org/10.1103/PhysRevLett. 121.091102.

[29] D. Radice and L. Dai, Eur. Phys. J. A 55 (2019), URL https://doi.org/10.1140/epja/i2019-12716-4.

[30] C. D. Capano, I. Tews, S. M. Brown, B. Margalit, S. De, S. Kumar, D. A. Brown, B. Krishnan, and S. Reddy, Nature News (2020), URL https://doi.org/10.1038/ s41550-020-1014-6.

[31] N. B. d'Etivaux, S. Guillot, J. Margueron, N. Webb, M. Catelan, and A. Reisenegger, Astrophys. J. 887, 48 (2019), URL https://doi.org/10.3847/1538-4357/ $\mathrm{ab} 4 \mathrm{f} 6 \mathrm{c}$.

[32] J.-L. Jiang, S.-P. Tang, D.-S. Shao, M.-Z. Han, Y.-J. Li, Y.-Z. Wang, Z.-P. Jin, Y.-Z. Fan, and D.-M. Wei, The Astrophysical Journal 885, 39 (2019), URL https: //doi.org/10.3847/1538-4357/ab44b2.

[33] T. Dietrich, M. W. Coughlin, P. T. Pang, M. Bulla, J. Heinzel, L. Issa, I. Tews, and S. Antier (2020), arXiv:2002.11355, URL https://arxiv.org/abs/2002. 11355.

[34] P. Landry and R. Essick, Phys. Rev. D 99, 084049 (2019), URL https://doi.org/10.1103/PhysRevD.99.084049.

[35] P. Landry, R. Essick, and K. Chatziioannou, Phys. Rev. D 101, 123007 (2020), URL https://doi.org/10.1103/ PhysRevD.101.123007.

[36] R. Essick, I. Tews, P. Landry, S. Reddy, and D. E. Holz (2020), arXiv:2004.07744, URL https://arxiv. 
org/abs/2004.07744.

[37] J.-L. Jiang, S.-P. Tang, Y.-Z. Wang, Y.-Z. Fan, and D.M. Wei, Astrophys. J. 892, 55 (2020), URL https:// doi.org/10.3847/1538-4357/ab77cf.

[38] J. M. Lattimer, Universe 5, 159 (2019), ISSN 2218-1997, URL http://dx.doi.org/10.3390/universe5070159.

[39] A. Ayriyan, D. Alvarez-Castillo, D. Blaschke, and H. Grigorian, Universe 5 (2019), ISSN 2218-1997, URL https://doi.org/10.3390/universe5020061.

[40] G. Raaijmakers et al., Astrophys. J. Lett. 893, L21 (2020), URL https://doi.org/10.3847/2041-8213/ ab822f.

[41] C. Raithel, F. Ozel, and D. Psaltis (2020), arXiv:2004.00656, URL https://arxiv.org/abs/ 2004.00656.

[42] A. W. Steiner, S. Gandolfi, F. J. Fattoyev, and W. G. Newton, Phys. Rev. C 91, 015804 (2015), URL https: //doi.org/10.1103/PhysRevC.91.015804.

[43] A. W. Steiner, J. M. Lattimer, and E. F. Brown, Astrophys. J. 722, 33 (2010), URL https://doi.org/10. 1088/0004-637X/722/1/33.

[44] A. W. Steiner and S. Gandolfi, Phys. Rev. Lett. 108, 081102 (2012), URL https://doi.org/10.1103/ PhysRevLett. 108.081102.

[45] J. S. Read, B. D. Lackey, B. J. Owen, and J. L. Friedman, Phys. Rev. D 79, 124032 (2009), 0812.2163, URL https: //doi.org/10.1103/PhysRevD.79.124032.

[46] J. M. Lattimer and A. W. Steiner, Eur. Phys. J. A 50, 40 (2014), URL https://doi.org/10.1140/epja/ i2014-14040-y.

[47] A. W. Steiner, J. M. Lattimer, and E. F. Brown, Astrophys. J. Lett. 765, 5 (2013), URL https://doi.org/10. 1088/2041-8205/765/1/L5.

[48] S. Greif, G. Raaijmakers, K. Hebeler, A. Schwenk, and A. Watts, Mon. Not. Roy. Astron. Soc. 485, 5363 (2019), 1812.08188, URL https://doi.org/10.1093/ mnras/stz654.

[49] A. W. Steiner, C. O. Heinke, S. Bogdanov, C. Li, W. C. G. Ho, A. Bahramian, and S. Han, Mon. Not. Roy. Astron. Soc. 476, 421 (2018), URL https://doi. org/10.1093/mnras/sty215.

[50] R. C. Tolman, Phys. Rev. 55, 364 (1939), URL https: //doi.org/10.1103/PhysRev.55.364.

[51] J. Oppenheimer and G. Volkoff, Phys. Rev. 55, 374 (1939), URL https://doi.org/10.1103/PhysRev.55. 374.

[52] K. Yagi and N. Yunes, Science 341, 365 (2013), URL https://doi.org/10.1126/science.1236462.

[53] A. W. Steiner, J. M. Lattimer, and E. F. Brown, Eur. Phys. J. A 52, 18 (2016), URL https://doi.org/10. 1140/epja/i2016-16018-1.
[54] S. Han and A. W. Steiner, Phys. Rev. D 99, 083014 (2019), URL https://doi.org/10.1103/PhysRevD.99. 083014.

[55] Z. Carson, K. Chatziioannou, C.-J. Haster, K. Yagi, and N. Yunes, Phys. Rev. D 99, 083016 (2019), URL https: //doi.org/10.1103/PhysRevD.99.083016.

[56] J. Nättilä, A. W. Steiner, J. J. E. Kajava, V. F. Suleimanov, and J. Poutanen, Astron. Astrophys. 591, A25 (2016), URL https://doi.org/10.1051/ 0004-6361/201527416.

[57] J. Nättilä, M. C. Miller, A. W. Steiner, J. J. E. Kajava, V. F. Suleimanov, and J. Poutanen, Astron. and Astrophys. 608, A31 (2017), URL https:/doi.org/10.1051/ 0004-6361/201731082.

[58] LIGO Scientific Collab. and Virgo Collab., Phys. Rev. X 9, 011001 (2019), URL https://doi.org/10.1103/ PhysRevX.9.011001.

[59] J. Lange, R. O'Shaughnessy, and M. Rizzo, arXiv:1805.10457 (2018), URL https://arxiv.org/ abs/1805.10457.

[60] R. Abbott et al. (LIGO Scientific, Virgo), Astrophys. J. Lett. 896, L44 (2020), URL https://doi.org/10.3847/ 2041-8213/ab960f.

[61] R. Essick and P. Landry (2020), arXiv:2007.01372, URL https://arxiv.org/abs/2007.01372.

[62] I. Tews, P. T. Pang, T. Dietrich, M. W. Coughlin, S. Antier, M. Bulla, J. Heinzel, and L. Issa (2020), arXiv:2007.06057, URL https://arxiv.org/abs/2007. 06057.

[63] D. Radice, A. Perego, F. Zappa, and S. Bernuzzi, Astrophys. J. Lett. 852, L29 (2018), URL https://doi.org/ 10.3847/2041-8213/aaa402.

[64] A. Bauswein and N. Stergioulas, Mon. Not. Roy. Astron. Soc. 471, 4956 (2017), 1702.02567, URL https://doi. org/10.1093/mnras/stx1983.

[65] K. Kiuchi, K. Kyutoku, M. Shibata, and K. Taniguchi, Astrophys. J. Lett. 876, L31 (2019), 1903.01466, URL https://doi.org/10.3847/2041-8213/ab1e45.

[66] M. Ruiz, S. L. Shapiro, and A. Tsokaros, Phys. Rev. D 97, 021501 (2018), URL https://doi.org/10.1103/ PhysRevD.97.021501.

[67] A. W. Steiner, bamr: Bayesian analysis of mass and radius observations (2014), Astrophysics Source Code Library, record ascl:1408.020, URL http://ascl.net/ 1408.020

[68] A. W. Steiner, O2scl: Object-oriented scientific computing library (2014), Astrophysics Source Code Library, record ascl:1408.019, URL http://ascl.net/1408.019.

[69] F. Frigo and S. G. Johnson, Fftw: Fastest fourier transform in the west (2012), FFTW: Fastest Fourier Transform in the West, record ascl:1201.015, URL http:// ascl.net/1201.015. 